\title{
HLA - Antigen and Haplotype Frequencies in Renal Transplant Recipients and Donors of Maharashtra (Western India)
}

\author{
Devaraj Prasanavar and U. Shankarkumar \\ HLA Department, Institute of Immunohaematology (ICMR), $13^{\text {th }}$ Floor, K.E.M.Hospital, \\ Parel, Mumbai 400 012, Maharahtra, India
}

\author{
KEYWORDS Renal patients; Maharashtra; HLA frequencies
}

\begin{abstract}
HLA antigen and haplotype frequencies were analysed in 217 renal transplant recipients and live related donors. It has been seen that the HLA - A and B antigen frequencies were not significantly different in two groups. Two and three locus haplotype analysis was also carried out in the renal transplant recipients and the donors to find out the most common haplotype. It was observed that two locus haplotypes HLA - A2 - B40; A9 - B35; A1 - B17; A19 - B35; A2 - DR2; A9 - DR6; B40 - DR2; were found to be common among recipients and donors of Maharashtra population, at HLA A and B; A and DR, as well as B and DR locus. Three locus haplotype analysis revealed that there were only two haplotypes A10-B8-DR4; A9-B35-DR6; were common in both donors and recipients. We have compared our results with other World and Indian population and have observed unique pattern of HLA phenotype and haplotype, which suggests that it is important to create a notional donor registry and organ-sharing network for Indian sub continent.
\end{abstract}

\section{INTRODUCTION}

Renal transplantation generally is the treatment of choice of patients with chronic renal failure. In the world the demand for renal transplant is about 60 patients per million population. There are around more than 1,50,000 patients suffering from kidney ailments in India. In Mumbai alone there are at least 1500-2000 patients who are on dialysis. Dialysis is an incomplete and long expensive treatment. On the other hand transplant gives the kidney a completely normal function. There are many centers, which do such transplants from live related donors. The success of such transplantation depends on the HLA. compatibility between recipients and donors. HLA locus is highly polymorphic so to find out matched donor and recipient is very difficult task.

Human leucocyte antigens are encoded by major histocompatibility complex (MHC) located on short arm of chromosome no 6. HLA molecules bind and present peptide to Tlymphocytes in cell mediated immune response and play a key role in shaping the T-cell repertoire on it and is also associated with allograft rejections. HLA antigens are inherited in a codominant manner from parents to the off springs. In the live related transplants, there are only $25 \%$ chances of that two sibs would be hundred percent identical, $50 \%$ chances would be that they will share one haplotype and $25 \%$ would be that they will not share any of the haplotype. when matched donor is not found in a nuclear family then extended search may be performed in the population to find out unrelated donors. Therefore the information about frequencies with which a particular HLA haplotype occur in a population is important. This information is required to calculate the probability to find out unrelated donors from a particular ethnic group.

Maharashtra the western state of India, lying between $74^{\circ}$ to $78^{\circ} \mathrm{E}$ longitude and $18^{\circ}-20^{\circ} \mathrm{N}$ latitude with the Mumbai as its capital city consist of 32 districts. Hindus form (81.94\%) majority of the population, while others are Muslims (8.4\%), Christians (1.42\%), Buddhists (6.48\%), Sikhs $(0.2 \%)$ and Jains (1.64\%) (Dikshit 1986).

\section{MATERIALS AND METHODS}

We have analyzed the HLA - A, B and DR phenotypes of 217 renal transplant recipients and their prospective live related donors referred to our Institute from 1996 - 2002. Blood samples from renal transplant recipients and donors were studied for HLA A,B and DR loci antigens. Their population specific details and genealogy were recorded in the precoded questionnaire. Five to ten milliliters of venous blood (in heparin 50IU/ ML) was collected in a sterile tube from each individual. The lymphocytes were isolated by density gradient centrifugation on histopaque (Boyum 1968). HLA A, B and DR locus antigens 
were identified by NIH two stage Microlymphocytoxicity assay (Terasaki and McClelland 1964) using T-cells for class I typing and B cells isolated by a miniature nylon wool column for class II with longer incubation period (Manikasundari et al 1984). A total of 190 anti HLA antisera were used for defining 17 specificities for HLA - A locus, 29 for HLA B locus antigens and 14 for HLA DR locus antigens. The antisera were commercial (Biotest, Geramany; Pelfreez, USA ; Behring, Germany) as well as indigenous (Shankarkumar et al. 1998) in origin. The typing tray included a minimum of three antisera for each supertypic specificity. The phenotype frequency, allele frequency and haplotype frequencies were calculated following the methods described by Baur and Daniloves (1980).They were used to measure differences between population and to compare our results with other population.

\section{RESULTS}

Allele frequency distributions of HLA-A, B and DR antigens in renal transplant recipients and donors are shown in tables (1, 2 and 3 ). Analyzing data the allele frequencies of various antigens at HLA A, B and DR locus we observed that they did not differ significantly in recipients and donors. Two locus haplotype (HLA A and $\mathrm{B}, \mathrm{B}$ and DR as well as A and DR) and three locus (HLA A, B and DR) extended haplotype analysis were done from both renal transplant recipients and donors (Tables 4, 5, 6 and 7). It was observed that HLA-A2-B40; A9-B35; A1-B17; A19-B35; A2-DR2; A9-DR6; B40-DR2; B40-DR2 were found to be common among recipients and donors of
Maharashtra population at HLA A and B, A and $\mathrm{DR}$, as well as B and DR loci. The occurrences of some of these common haplotypes in between the incidence of these antigens were almost equal in both the groups. Three locus haplotype analysis revealed that there were only two haplotypes A10-B8-DR4; A9-B35-DR6 common in both donors and recipients.

\section{DISCUSSION}

We have done HLA class I and II typing for 217 live related renal transplant. Among them $29.2 \%$ were siblings $.65 \%$ were parents $\mathrm{V} / \mathrm{s}$ offspring, $2.7 \%$ were spousal donors, $3.1 \%$ were other donors like cousins or uncles. In the present study we have not seen any difference between recipients and donors at individual antigen basis. HLA A, B and DR antigens did not differ from one another among the donors and the recipients. Both the donors and the recipients population were found to be in the genetic equilibrium.

HLA antigen frequencies from the present study were compared with other Indian population like North Indians from U. P. and South Indians from Tamil Nadu. Allele frequency of HLA-A3, A19, B35, B40, DR2, DR6 were increased in our population as compared to North Indian from U. P (Agarwal et al. 2001). Where as HLA-A10, A11, B8, B15, DR1, DR3, DR7 were decreased. It was also observed that the haplotypes A1-B17, A2-B40, A9-B35, A19-B35, A2-DR2, A9-DR6, B40-DR2 were increased, while the haplotype A11-B35 was reduced in our population compared to South Indians (Pitchappan et al. 1984).

Previous studies show that haplotypes

Table 1: Allele frequencies of HLA - A locus in renal transplant recipients and donors of western India compared with other Indian and world population

\begin{tabular}{|c|c|c|c|c|c|c|c|c|c|c|c|c|}
\hline \multirow[b]{2}{*}{ Antigen } & \multicolumn{5}{|c|}{ Patient } & \multicolumn{7}{|c|}{ Donor } \\
\hline & $W I$ & $U P$ & $A F A$ & $C A U$ & $H I S$ & $W I$ & $U P$ & $N I$ & $S I$ & $A F A$ & $C A U$ & $H I S$ \\
\hline 1 & 0.15 & 0.19 & 0.09 & 0.28 & 0.12 & 0.14 & 0.2 & 0.27 & 44.17 & 0.09 & 0.29 & 0.13 \\
\hline 2 & 0.19 & 0.21 & 0.32 & 0.47 & 0.46 & 0.17 & 0.22 & 0.23 & 36.67 & 0.37 & 0.5 & 0.49 \\
\hline 3 & 0.07 & 0.1 & 0.15 & 0.25 & 0.15 & 0.06 & 0.22 & 0.17 & 14.14 & 0.16 & 0.27 & 0.17 \\
\hline 9 & 0.21 & 0.13 & 0.02 & 0.1 & 0.02 & 0.2 & 0.14 & 0.27 & 28.74 & 0 & 0 & 0.01 \\
\hline 10 & 0.06 & 0.12 & 0.01 & 0.01 & 0.01 & 0.04 & 0.1 & 10.5 & 6.7 & 0.01 & 0 & 0 \\
\hline 11 & 0.06 & 0.19 & 0.03 & 0.12 & 0.08 & 0.08 & 0.16 & 25.7 & 28.33 & 0.03 & 0.12 & 0.11 \\
\hline 19 & 0.15 & 0.04 & 0.04 & 0.01 & 0.12 & 0.17 & 0.06 & 33.5 & 6.25 & 0.01 & 0 & 0.01 \\
\hline 28 & 0.06 & 0.07 & 0.02 & 0.07 & 0.14 & 0.06 & 0.06 & 15.2 & 9.17 & 0.12 & 0.06 & 0.14 \\
\hline 36 & 0.01 & 0.03 & 0.05 & 0 & 0.01 & 0.06 & 0.03 & 0 & 0 & 0.04 & 0 & 0 \\
\hline A- & 0.05 & 0.19 & 0.17 & 0.16 & 0.16 & 0.06 & 0.18 & 0 & 0 & 0.09 & 0.09 & 0.1 \\
\hline
\end{tabular}

WI=Western India,UP=Uttar Pradesh, AFA=African- American, CAU=Caucasion,HIS=Hispanics. 
HLA - ANTIGEN AND HAPLOTYPE FREQUENCIES IN RENAL TRANSPLANT RECIPIENTS

Table 2: Allele frequencies of HLA - B locus in renal transplant recipients and donors of western India compared with other Indian and world population

\begin{tabular}{|c|c|c|c|c|c|c|c|c|c|c|c|c|}
\hline \multirow[b]{2}{*}{ Antigen } & \multicolumn{5}{|c|}{ Patient } & \multicolumn{7}{|c|}{ Donor } \\
\hline & $W I$ & $U P$ & $A F A$ & $C A U$ & $H I S$ & $W I$ & $U P$ & $N I$ & $S I$ & $A F A$ & $C A U$ & $H I S$ \\
\hline 5 & 0.11 & 0.11 & 0.02 & 0.01 & 0.02 & 0.12 & 0.13 & 0.3 & 28.75 & 0.01 & 0.01 & 0 \\
\hline 7 & 0.13 & 0.16 & 0.17 & 0.19 & 0.11 & 0.14 & 0.17 & 0.13 & 5.83 & 0.19 & 0.25 & 0.1 \\
\hline 8 & 0.05 & 0.08 & 0.08 & 0.22 & 0.07 & 0.05 & 0.08 & 0.09 & 5.63 & 0.07 & 0.21 & 0 \\
\hline 12 & 0.07 & 0.05 & 0.02 & 0.01 & 0.01 & 0.06 & 0.05 & 0.17 & 10 & 0 & 0 & 0 \\
\hline 13 & 0.03 & 0.05 & 0.02 & 0.04 & 0.02 & 0.02 & 0.05 & 0.15 & 7.5 & 0.01 & 0.05 & 0 \\
\hline 14 & 0.08 & 0.01 & 0.05 & 0.07 & 0.08 & 0 & 0.01 & 0 & 3.75 & 0.04 & 0.06 & 0 \\
\hline 15 & 0 & 0.04 & 0.1 & 0.01 & 0.01 & 0.06 & 0.05 & 0.14 & 7.92 & 0.01 & 0 & 0 \\
\hline 16 & 0.02 & 0.03 & 0.06 & 0.01 & 0.02 & 0 & 0.03 & 0.02 & 0 & 0 & 0 & 0 \\
\hline 17 & 0.01 & 0.05 & 0.06 & 0.02 & 0.02 & 0.09 & 0.05 & 0.15 & 20.42 & 0.06 & 0.01 & 0 \\
\hline 18 & 0.01 & 0.04 & 0.01 & 0.1 & 0.11 & 0.01 & 0.06 & 0.05 & 1.67 & 0.06 & 0.08 & 0 \\
\hline 21 & 0.03 & 0.07 & 0.01 & 0.01 & 0.01 & 0.01 & 0.05 & 0.07 & 0 & 0 & 0 & 0 \\
\hline 22 & 0.03 & 0.05 & 0.02 & 0.01 & 0.01 & 0 & 0.04 & 0.06 & 10 & 0 & 0.01 & 0 \\
\hline 27 & 0.05 & 0.06 & 0.15 & 0.09 & 0.05 & 0.03 & 0.05 & 0.06 & 2.08 & 0.02 & 0.08 & 0 \\
\hline 35 & 0.18 & 0.07 & 0.15 & 0.17 & 0.26 & 0.16 & 0.09 & 0.27 & 18.75 & 0.15 & 0.02 & 0.2 \\
\hline 37 & 0.02 & 0.01 & 0.01 & 0.29 & 0.02 & 0.02 & 0.01 & 0 & 0 & 0.01 & 0.03 & 0 \\
\hline 40 & 0.16 & 0.05 & 0.01 & 0.02 & 0.02 & 0.16 & 0.05 & 0.23 & 20.42 & 0 & 0.01 & 0 \\
\hline 47 & 0 & 0.01 & 0 & 0.01 & 0 & 0 & 0 & 0 & 0 & 0 & 0 & 0 \\
\hline 53 & 0.02 & 0.03 & 0.22 & 0.02 & 0.04 & 0 & 0.04 & 0 & 0 & 0.19 & 0.01 & 0 \\
\hline B- & 0 & 0.21 & 0.14 & 0.11 & 0.13 & 0 & 0.19 & 0 & 0 & 0.07 & 0.05 & 0.06 \\
\hline
\end{tabular}

WI=Western India,UP=Uttar Pradesh, AFA=African- American, CAU=Caucasion, HIS=Hispanics

Table 3: Allele frequencies of HLA - DR locus in renal transplant recipients and donors of western India compared with other Indian and world population

\begin{tabular}{|c|c|c|c|c|c|c|c|c|c|c|c|c|}
\hline \multirow[b]{2}{*}{ Antigen } & \multicolumn{5}{|c|}{ Patient } & \multicolumn{7}{|c|}{ Donor } \\
\hline & $W I$ & $U P$ & $A F A$ & $C A U$ & $H I S$ & $W I$ & $U P$ & $N I$ & $S I$ & $A F A$ & $C A U$ & $H I S$ \\
\hline 1 & 0.02 & 0.27 & 0.10 & 0.17 & 0.14 & 0.02 & 0.27 & 0.14 & 0.00 & 0.13 & 0.18 & 0.16 \\
\hline 2 & 0.29 & 0.20 & 0.20 & 0.16 & 0.14 & 0.30 & 0.20 & 0.47 & 0.00 & 0.20 & 0.22 & 0.15 \\
\hline 3 & 0.08 & 0.17 & 0.25 & 0.25 & 0.16 & 0.07 & 0.17 & 0.26 & 0.00 & 0.22 & 0.21 & 0.13 \\
\hline 4 & 0.10 & 0.11 & 0.10 & 0.36 & 0.39 & 0.08 & 0.11 & 0.26 & 0.00 & 0.09 & 0.31 & 0.37 \\
\hline 5 & 0.10 & 0.16 & 0.10 & 0.07 & 0.09 & 0.06 & 0.16 & 0.23 & 0.00 & 0.88 & 0.06 & 0.08 \\
\hline 6 & 0.20 & 0.06 & 0.18 & 0.15 & 0.12 & 0.23 & 0.06 & 0.18 & 0.00 & 0.17 & 0.15 & 0.15 \\
\hline 7 & 0.10 & 0.26 & 0.18 & 0.16 & 0.15 & 0.11 & 0.26 & 0.22 & 0.00 & 0.19 & 0.24 & 0.19 \\
\hline 9 & 0.01 & 0.06 & 0.07 & 0.03 & 0.03 & 0.00 & 0.06 & 0.03 & 0.00 & 0.52 & 0.22 & 0.03 \\
\hline 10 & 0.04 & 0.06 & 0.03 & 0.02 & 0.03 & 0.06 & 0.06 & 0.02 & 0.00 & 0.04 & 0.02 & 0.02 \\
\hline 13 & 0.01 & 0.08 & 0.15 & 0.11 & 0.09 & 0.00 & 0.08 & 0.00 & 0.00 & 0.21 & 0.13 & 0.12 \\
\hline Blank & 0.00 & 0.25 & 0.24 & 0.25 & 0.29 & 0.00 & 0.25 & 0.00 & 0.00 & 0.12 & 0.13 & 0.14 \\
\hline
\end{tabular}

WI=Western India,UP=Uttar Pradesh, AFA=African-American,CAU=Caucasion,HIS=Hispanics

Table 4: Most Common HLA-A\&B Haplotypes of renal transplant recipients and donors from Western India.

\begin{tabular}{|c|c|c|c|c|c|c|c|}
\hline \multirow[b]{2}{*}{ A } & \multirow[b]{2}{*}{$B$} & \multicolumn{2}{|c|}{ Patient } & \multirow[b]{2}{*}{$A$} & \multirow[b]{2}{*}{$B$} & \multicolumn{2}{|c|}{ Donor } \\
\hline & & $W I$ & $U P$ & & & $W I$ & $U P$ \\
\hline 1 & 7 & 230 & 27 & 1 & 7 & 321 & 0 \\
\hline 1 & 17 & 253 & 0 & 1 & 17 & 344 & 0 \\
\hline 1 & 35 & 230 & 0 & 1 & 35 & 161 & 0 \\
\hline 2 & 7 & 253 & 171 & 2 & 7 & 367 & 0 \\
\hline 2 & 27 & 138 & 0 & 2 & 27 & 92 & 0 \\
\hline 2 & 35 & 161 & 0 & 2 & 35 & 505 & 0 \\
\hline 2 & 40 & 806 & 0 & 2 & 40 & 757 & 0 \\
\hline 9 & 35 & 530 & 0 & 9 & 35 & 459 & 0 \\
\hline 9 & 7 & 230 & 0 & 9 & 7 & 367 & 126 \\
\hline 10 & 8 & 346 & 0 & 10 & 8 & 183 & 0 \\
\hline 19 & 35 & 300 & 0 & 19 & 35 & 367 & 0 \\
\hline 19 & 40 & 138 & 0 & 19 & 40 & 161 & 0 \\
\hline
\end{tabular}

WI=Western India,UP=Uttar Pradesh. 
Table 5: Most common HLA-A\&DR Haplotypes of renal transplant recipients and donors of Western India

\begin{tabular}{lrrrrrrrr}
\hline & & \multicolumn{2}{c}{ Patient } & & & \multicolumn{2}{c}{ Donor } \\
\cline { 2 - 3 } \cline { 6 - 8 }$A$ & $D R$ & $W I$ & $U P$ & & & DR & WI & UP \\
\hline 2 & 7 & 119 & 260 & 1 & 7 & 116 & 79 \\
2 & 2 & 833 & 0 & 2 & 7 & 116 & 0 \\
2 & 6 & 238 & 0 & 2 & 2 & 814 & 0 \\
3 & 2 & 119 & 209 & 2 & 6 & 349 & 0 \\
9 & 6 & 595 & 65 & 3 & 2 & 116 & 287 \\
9 & 10 & 238 & 0 & 9 & 6 & 581 & 0 \\
9 & 4 & 238 & 0 & 9 & 4 & 233 & 0 \\
9 & 2 & 357.1 & 149 & 10 & 2 & 232 & 13 \\
10 & 6 & 119 & 96 & 10 & 6 & 116 & 56 \\
19 & 7 & 357.1 & 0 & 11 & 1 & 116 & 201 \\
11 & 1 & 119 & 239 & 19 & 7 & 349 & 147 \\
\hline
\end{tabular}

Table 6: Most common HLA-B\&DR Haplotypes of renal Transplant recipients and donors of Western India

\begin{tabular}{|c|c|c|c|c|c|c|c|}
\hline \multirow[b]{2}{*}{ A } & \multirow[b]{2}{*}{$D R$} & \multicolumn{2}{|c|}{ Patient } & \multirow[b]{2}{*}{$B$} & \multirow[b]{2}{*}{$D R$} & \multicolumn{2}{|c|}{ Donor } \\
\hline & & $W I$ & $U P$ & & & $W I$ & $U P$ \\
\hline 6 & 119.0 & 4 & 0 & 5 & 6 & 116 & 0 \\
\hline 7 & 4 & 238 & 0 & 7 & 4 & 116 & 0 \\
\hline 7 & 5 & 119.04 & 0 & 7 & 5 & 116 & 0 \\
\hline 7 & 6 & 119.04 & 0 & 7 & 6 & 116 & 0 \\
\hline 8 & 2 & 119.04 & 0 & 8 & 2 & 116 & 0 \\
\hline 8 & 3 & 119 & 0 & 8 & 3 & 116 & 0 \\
\hline 12 & 7 & 119 & 0 & 12 & 7 & 116 & 0 \\
\hline 15 & 6 & 238 & 0 & 15 & 6 & 116 & 0 \\
\hline 17 & 2 & 238 & 0 & 17 & 2 & 238 & 0 \\
\hline 22 & 7 & 119 & 0 & 22 & 7 & 116 & 0 \\
\hline 22 & 6 & 119 & 0 & 22 & 6 & 116 & 0 \\
\hline 27 & 3 & 119 & 0 & 27 & 3 & 116 & 0 \\
\hline 35 & 10 & 119 & 0 & 35 & 10 & 116 & 0 \\
\hline 35 & 4 & 238 & 0 & 35 & 4 & 238 & 0 \\
\hline 40 & 2 & 357 & 0 & 40 & 2 & 348 & 0 \\
\hline 40 & 6 & 3 & 0 & 40 & 6 & 465 & 0 \\
\hline 53 & 7 & 119 & 0 & 53 & 7 & 116 & 0 \\
\hline
\end{tabular}

Table 7: Most common HLA-A, B \& DR Haplotypes of renal transplant recipients and donors of Western India

\begin{tabular}{lrrrrrrrrr}
\hline & & \multicolumn{2}{c}{ Patient } & & & \multicolumn{2}{c}{ Donor } \\
\cline { 3 - 9 }$A$ & $B$ & $D R$ & $W I$ & $U P$ & $A$ & $B$ & $D R$ & WI & UP \\
\hline 2 & 40 & 2 & 119 & 0 & 2 & 40 & 2 & 119 & 0 \\
2 & 27 & 6 & 119 & 0 & 2 & 27 & 6 & 119 & 0 \\
9 & 35 & 6 & 238 & 0 & 9 & 35 & 6 & 119 & 0 \\
10 & 8 & 4 & 238 & 0 & 10 & 8 & 4 & 238 & 0 \\
9 & 40 & 6 & 119 & 0 & 9 & 40 & 6 & 238 & 0 \\
1 & 9 & 35 & 2 & 0 & 19 & 35 & 2 & 119 & 0
\end{tabular}

among population/caste groups were different. HLA A10-B8, A19-B12, A9-B15 and A3-B5 are common in Maharashtra Brahmin (Shankarkumar et al. 2000), while the haplotypes A3-B35, A19B15 and A11-B40 are unique in Marathi speaking Hindu population (Shankarkumar et al 1999), A9B5, A10-B8 and A1-B17 in Maratha (Shankarkumar et al. 2001) where as A1-B17, A10-B8, A19B12 and A9-B35 are common Indian haplotypes
(Ghosh et al. 2002). The antigen frequencies were compared with donor of different ethnic groups (i. e. African-Americans, Caucasians, Oriental and Hispanics) from United Nation of Organ Sharing (UNOC) renal registry data at HLA A, B and DR loci (Lefell et al 1994; Zachary et al 1996). Two and Three locus haplotype analyses was carried out and it has been seen that A1-B17, A2-B40, A9-B35, A19-B35, A2-DR2, A9-DR6 and B40-DR2 
at two locus haplotype were found to be common haplotypes among recipients and donors of Maharashtra Population. Three-locus haplotype analysis revealed that, there were only two haplotypes A10-B8-DR4, A9-B35-DR6 were common in both the donors and recipients. While other haplotypes were rare and occurred with low frequencies.

The data presented in this study for the HLA allele and haplotype frequencies of Maharashtra population is the first report for renal transplant recipients and donors from western part of the country. While the accuracy of the frequency estimated diminishes for vary rare haplotypes, comparison with similar data from other sources indicate that this data is over all reasonable estimates of the haplotype frequencies that may be applied for various purposes, such as predictive modeling in transplantation UNOS renal transplant registry demonstrates that the large disparity exists between African-American and Caucasoid in the HLA frequencies. If haplotype identical cadaver renal transplant is required then large registries are to be created for end stage renal disease patients. Certain haplotypes particularly two loci and three loci are common to some of the racial groups which reduces the registry size. However this does not hold true for our population, as Indian population does not appear to share the phenotypes with other racial and ethnic groups within the country and also with the world population. Hence, there is a need to compare haplotype frequencies among the populations from various geographical regions of India. This would be important for creating a national registry and organ - sharing network for Indian population both for Indians living in India and also abroad.

\section{REFERENCES}

Agarwal S, Singh AK, Sharma RK 2001. HLA gene and haplotype frequency in renal transplant recipients and donors of Utter pradesh (North India). Indian Journal of Nephorology, (Suppl.) 188.

Baur MP, Daniloves JA 1980. Population analysis of HLA A, B, C, DR and other genetic markers. In: PI Terasaki (Ed.): Histocompitibility testing 1980. Los Angels: UCLA Press. Pp 955 - 1210

Dikshit KR 1986. Maharashtra in Maps. Govt. of
Maharashtra (Eds.) India.

Ghosh K, Shankarkumar U, Mohanty D 2002. Common human leucocyte antigen haplotype in Indians - its implications in finding unrelated compatible bone marrow donors Transfusion Medicine, 12: 43-48

Lefell, MS, Steinberg Bias WB, Machan, $\mathrm{CH}$, Zachary AA 1994. The distribution of antigens and phenotypes among donors and patients in the UNOS registry. Transplantation, 10: 1119-1130.

Manikasundari M, Selvaraj P, Pitchappan R.M 1984. Studies on T cells of the Lizard calotes versicolor: Adherent and non-adherent population of the spleen. Dev Comp Immunol, 8: 367-374.

Mattiuz PL, Indr D, Piazza A, Ceppellini R, Bodner WF 1970. New approaches to the population genetics and segregation analysis of HLA system. In: PI Terasaki (Ed.): Histocompatibility Testing. Copenhagen: Munksgoord. Pp 193-205.

Mehra NK, Taneja V, Kailas S, Raizada N, Vaidya MC 1986. Distribution of HLA antigen in samples of North Indian Hindu population. Tissue Antigens, 27 : 64-74.

Nei M 1972. Genetic distance between populations: American Naturalist, 106: 283-292.

Piazza A 1975. Haplotype and linkage disequilibrium from three locus phenotype In: Histocomapatibility testing 1975. Copenhagen: Munksgoord. Pp 923.

Pitchappan RM, Kakkanaiah VN, Rajashekhar R, Arulraj N, Muthukaruppan VR 1984. HLA antigen in south Indians : major group of Tamilnadu. Tissue Antigens, 24: 1906

Shankarkumar U 1998. HLA system and organ transplantation National Kidney Foundation (India), 19: 4-5.

Shankarkumar U, Ghosh K, Mohanty D 2001. HLA antigen distribution in Maratha community from Mumbai, Maharashtra India. Int J Hum Genet, 3: 173-177.

Shankarkumar U, Gupte SC, Gupte SS, Padnekar SV, Ghosh K, Mohanty D 1998. Frequency and potential application of HLA antibodies from pregnant women in Mumbai $J$ Biosci, 23: 601-604.

Shankarkumar U, Padnekar SV, Gupte S, Ghosh K, Mohanty D 1999. HLA antigen distribution in Marathi speaking Hindu population from Mumbai, Maharashtra. J Hum Ecol, 10: 367-372.

Shankarkumar U, Ghosh K, Mohanty D 2000. HLA class I antigen profile among Brahmin and related caste groups form Mumbai Maharashtra, India. Ind $J$ of Hum Genet, 6: 21-28.

Shankarkumar U 2001. HLA matching and problem related to cadaveric renal transplantation in India. Ind J Med Sci, 55:417-420.

Zachary AA, Steinberg AG, Bias WB, Lefell MS 1996.The frequencies of HLA alleles and haplotypes and their distribution among the donors in a renal transplant patients in the UNOS registry Transplantation, 62: 273 